\title{
Consistency checks of different $\pi \pi$ scattering data sets using forward dispersion relations
}

\author{
José R. Peláez \\ Dept. Física Teórica II. Universidad Complutense, 28040-Madrid, Spain
}

\begin{abstract}
We review our evaluation of forward dispersion relations for direct fits to the different, and often conflicting, $\pi \pi$ scattering experimental analyses. We find that some of the most commonly used data sets do not satisfy these constraints by several standard deviations. We also provide a consistent $\pi \pi$ amplitude by improving a global fit to data with these dispersion relations.
\end{abstract}

Keywords: mesons, pions, scattering, dispersion relations

PACS: $12.39 . \mathrm{Fe}, 13.75 . \mathrm{Lb}, 11.55 . \mathrm{Jy}, 11.80 . \mathrm{Et}$

A precise knowledge of the $\pi \pi$ scattering amplitude provides crucial tests for Chiral Perturbation Theory (ChPT), as well as information on light meson spectroscopy, pionic atom decays and $\mathrm{CP}$ violation in kaons. However, there are several $\pi \pi$ scattering data sets in the literature, in conflict among themselves, even within the same experiment. The reason is that the data are extracted from other reactions and thus with large theoretical and systematic uncertainties. Here we review our recent works [1, 2, 3] where we checked dispersion relations on the different sets of data and provided simple parameterizations of $\pi \pi$ scattering amplitudes consistent with such requirements.

\section{Fits to different sets of data}

We first consider fits to data [3] for the S0, S2, P waves, below $s^{1 / 2} \lesssim 1 \mathrm{GeV}$. We parameterize the phase shifts, $\delta(s)$, taking into account the analytic properties, zeros and poles of the amplitude, in a conformal expansion of $\cot \delta(s)$.

The $\mathrm{P}$ wave, up to $\simeq 1 \mathrm{GeV}$, comes from the following fit to the pion form factor [4]

$$
\begin{aligned}
& \cot \delta_{1}(s)=\frac{s^{1 / 2}}{2 k^{3}}\left(M_{\rho}^{2}-s\right)\left\{B_{0}+B_{1} \frac{\sqrt{s}-\sqrt{s_{0}-s}}{\sqrt{s}+\sqrt{s_{0}-s}}\right\} ; \quad s_{0}^{1 / 2}=1.05 \mathrm{GeV} . \\
& B_{0}=1.069 \pm 0.011, \quad B_{1}=0.13 \pm 0.05, \quad M_{\rho}=773.6 \pm 0.9
\end{aligned}
$$

( $s_{0}$ is the point where inelasticity begins to be nonnegligible). The fit is seen in Fig.1a.

For the S2 wave at low energies, we first fix the Adler zero at $z_{2}=M_{\pi}$ and fit only the low energy data, $s^{1 / 2}<1.0 \mathrm{GeV}$; later on we allow $z_{2}$ to vary. We have

$$
\begin{aligned}
& \cot \delta_{0}^{(2)}(s)=\frac{s^{1 / 2}}{2 k} \frac{M_{\pi}^{2}}{s-2 z_{2}^{2}}\left\{B_{0}+B_{1} \frac{\sqrt{s}-\sqrt{s_{0}-s}}{\sqrt{s}+\sqrt{s_{0}-s}}\right\}, \quad s_{0}^{1 / 2}=1.05 \mathrm{GeV} \\
& B_{0}=-80.4 \pm 2.8, \quad B_{1}=-73.6 \pm 12.6
\end{aligned}
$$



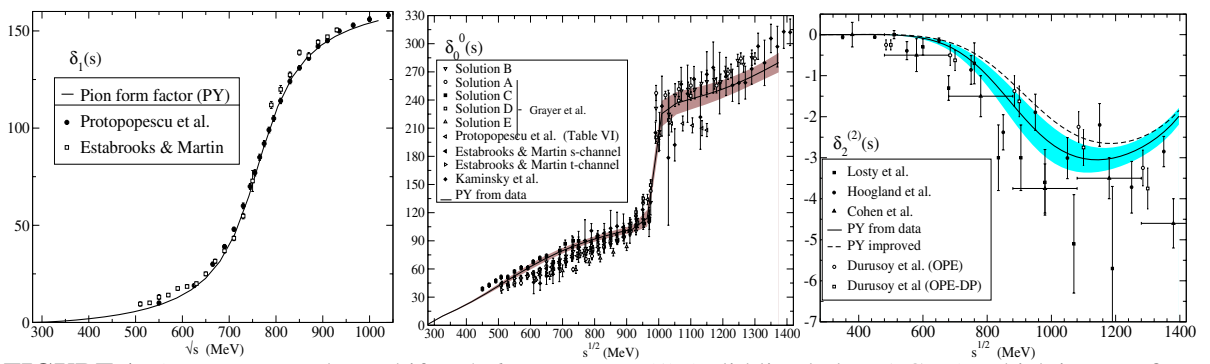

FIGURE 1. a) $\mathrm{P}$ wave $\pi \pi$ phase shifts $[5,6]$, versus Eq.(1) (solid line below $1 \mathrm{GeV}$ ), which is not a fit to these data, but to the pion form factor [4]. The uncertainty is the line thickness. Above $1 \mathrm{GeV}$, the dotted line and error are as in [3]. b) S0 phase shifts and error band as given by Eq.(4) below $1 \mathrm{GeV}$, and from [3] above. The $K_{l 4}$ and $K_{2 \pi}$ decay data are not shown. (see our [3] for details). c) Continuous line: The fit to $I=2, D$-wave phase shift data. Broken line: fit improved with dispersion relations. The experimental points are from [8].

Except for the very reliable $K_{l 4}$ and $K \rightarrow 2 \pi$ decay experiments [7], that we always fit, the S0 data is very confusing and thus we have adopted two approaches. In the first method, the "global fit", we fit averaged phase shift data between $0.81 \mathrm{GeV} \leq s^{1 / 2} \leq$ $0.97 \mathrm{GeV}$, (where the experiments agree within $1.5 \sigma$ ), composing their errors carefully. The fit, shown in Fig.1.b, is valid for $s^{1 / 2} \leq 0.95 \mathrm{GeV}$, and with the Adler zero fixed at $z_{0}=M_{\pi}$, corresponds to

$$
\begin{aligned}
& \cot \delta_{0}^{(0)}(s)=\frac{s^{1 / 2}}{2 k} \frac{M_{\pi}^{2}}{s-\frac{1}{2} z_{0}^{2}} \frac{M_{\mu}^{2}-s}{M_{\mu}^{2}}\left\{B_{0}+B_{1} \frac{\sqrt{s}-\sqrt{s_{0}-s}}{\sqrt{s}+\sqrt{s_{0}-s}}\right\}, \\
& B_{0}=y-x ; \quad B_{1}=6.62-2.59 x ; \quad y=21.04 \pm 0.70, \quad x=0 \pm 2.6 .
\end{aligned}
$$

The second method is to fit only $K_{l 4}$ and $K \rightarrow 2 \pi$ data, or to add to them the data from the various experimental analyses separately. The results can be found in Table 1.

The D2 inelasticity is negligible below $4 M_{\rho}^{2}$. A pole term is needed since the data [8] gives a small negative phase above $\sim 500 \mathrm{MeV}$, but the Froissart-Gribov representation, yields [9] a positive scattering length, $a_{2}^{(2)}=(2.72 \pm 0.36) \times 10^{-4} M_{\pi}^{-5}$, which is included in the fit. Also, the inflection seen in data around $1 \mathrm{GeV}$ asks for a third order conformal expansion. So we write

$$
\cot \delta_{2}^{(2)}(s)=\frac{s^{1 / 2}}{2 k^{5}}\left\{B_{0}+B_{1} w(s)+B_{2} w(s)^{2}\right\} \frac{M_{\pi}^{4} s}{4\left(M_{\pi}^{2}+\Delta^{2}\right)-s}, w(s)=\frac{\sqrt{s}-\sqrt{s_{0}-s}}{\sqrt{s}+\sqrt{s_{0}-s}} .
$$

And we find $B_{0}=(2.4 \pm 0.3) \times 10^{3}, B_{1}=(7.8 \pm 0.8) \times 10^{3}, B_{2}=(23.7 \pm 3.8) \times 10^{3}$, $\Delta=196 \pm 20 \mathrm{MeV}$. The fit is shown in Fig.1.c.

For brevity, we do not discuss here the D0 and $\mathrm{F}$ waves as they do not present special features, nor the intermediate energy region $1 \mathrm{GeV} \geq s^{1 / 2} \geq 1.42 \mathrm{GeV}$, but a detailed account of their parameterizations can be found in [3].

We also need the imaginary part of the scattering amplitude at $s^{1 / 2} \geq 1.42 \mathrm{GeV}$, that we take from a Regge fit to experimental data ( see [2] for details and the slightly improved rho residue of [3]), shown in Fig.2. As discussed in [2, 3], standard Regge factorization describes experiment $[10,2]$ and is consistent with crossing sum rules. 

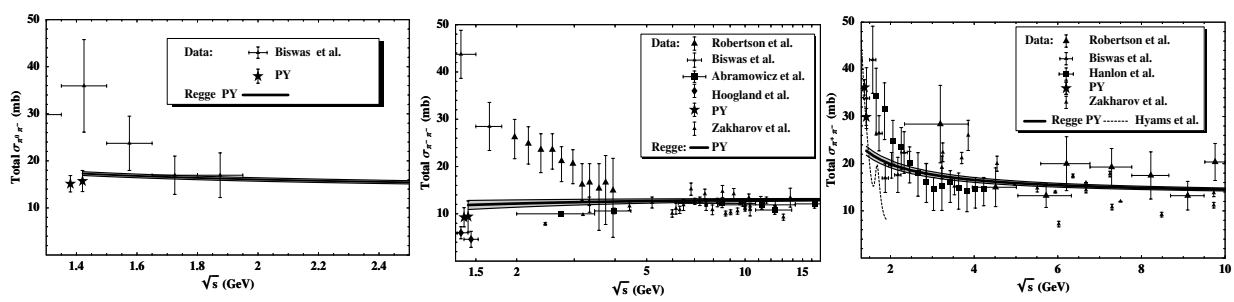

FIGURE 2 The $\pi \pi$ cross sections. Experimental points from [10]. The stars at 1.38 and $1.42 \mathrm{GeV}$ (PY) are from the phase shift analysis of experimental data given in [3]. Continuous lines, from $1.42 \mathrm{GeV}$ (PY): Regge formula, with parameters as in [2] (the gray bands cover the uncertainties). Below $2 \mathrm{GeV}$, the dotted line corresponds to the $\pi^{+} \pi^{-}$cross section from the Cern-Munich analysis of Hyams et al.[6]

\begin{tabular}{|c|c|c|c|c|c|}
\hline & $B_{0}$ & $B_{1}$ & $M_{\mu}(\mathrm{MeV})$ & $\begin{array}{c}I_{t}=1 \\
\chi^{2} / \text { d.o.f. }\end{array}$ & $\begin{array}{c}\pi^{0} \pi^{0} \\
\chi^{2} / \text { d.o.f. }\end{array}$ \\
\hline PY, Eq.(4) & 21.04 & 6.62 & $782 \pm 24$ & 0.3 & 3.5 \\
\hline$K$ decay only & $18.5 \pm 1.7$ & $\equiv 0$ & $766 \pm 95$ & 0.2 & 1.8 \\
\hline K decay + Grayer B [6] & $22.7 \pm 1.6$ & $12.3 \pm 3.7$ & $858 \pm 15$ & 1.0 & 2.7 \\
\hline K decay +Grayer C [6] & $16.8 \pm 0.85$ & $-0.34 \pm 2.34$ & $787 \pm 9$ & 0.4 & 1.0 \\
\hline K decay + Grayer E [6] & $21.5 \pm 3.6$ & $12.5 \pm 7.6$ & $1084 \pm 110$ & 2.1 & 0.5 \\
\hline K decay + Kamisnki [6] & $27.5 \pm 3.0$ & $21.5 \pm 7.4$ & $789 \pm 18$ & 0.3 & 5.0 \\
\hline K decay + Grayer A [6] & $28.1 \pm 1.1$ & $26.4 \pm 2.8$ & $866 \pm 6$ & 2.0 & 7.9 \\
\hline K decay+ EM, s-channel [6] & $29.8 \pm 1.3$ & $25.1 \pm 3.3$ & $811 \pm 7$ & 1.0 & 9.1 \\
\hline K decay+ EM, t-channel [6] & $29.3 \pm 1.4$ & $26.9 \pm 3.4$ & $829 \pm 6$ & 1.2 & 10.1 \\
\hline K decay+Protopopescu VI [5] & $27.0 \pm 1.7$ & $22.0 \pm 4.1$ & $855 \pm 10$ & 1.2 & 5.8 \\
\hline K decay+Protopopescu XII [5] & $25.5 \pm 1.7$ & $18.5 \pm 4.1$ & $866 \pm 14$ & 1.2 & 6.3 \\
\hline K decay+ Protopopescu 3 [5] & $27.1 \pm 2.3$ & $23.8 \pm 5.0$ & $913 \pm 18$ & 1.8 & 4.2 \\
\hline
\end{tabular}

TABLE 1 Fits to K decays and different data sets of S0 phase shifts. PY is our global fit, Eq.(4), and its $B_{0}$ and $B_{1}$ uncorrelated uncertainties can be obtained from Eq.(4). Note that many of these sets have very large $\chi^{2} /$ d.o.f. for the $I_{t}=1$ and $\pi^{0} \pi^{0}$ dispersion relations below $950 \mathrm{MeV}$. Let us also remark that some of them (like that of Sol. E) have a relatively small $\chi^{2} /$ d.o.f. just because it has huge uncertainties in its parameters. Ideally one would require a low $\chi^{2} /$ d.o.f. with small uncertainties in the parameters.

\section{Checking and improving amplitudes with forward dispersion relations}

Let us study how well the previous fits to data satisfy three independent scattering amplitudes. We choose the $t$-symmetric or antisymmetric combinations, that form a complete set: $F_{00} \equiv F\left(\pi^{0} \pi^{0} \rightarrow \pi^{0} \pi^{0}\right), F_{0+} \equiv F\left(\pi^{0} \pi^{+} \rightarrow \pi^{0} \pi^{+}\right)$, and the $t$ channel isospin one amplitude, $F^{\left(I_{t}=1\right)}$. Final uncertainties are small for the two first, since they depend only on two isospin states, and their imaginary parts are sums of positive terms. Thus, we find two dispersion relations by choosing either $F=F_{00}$ or $F=F_{0+}$ in

$$
\operatorname{Re} F(s)-F\left(4 M_{\pi}^{2}\right)=\frac{s\left(s-4 M_{\pi}^{2}\right)}{\pi} \text { P.P. } \int_{4 M_{\pi}^{2}}^{\infty} \mathrm{d} s^{\prime} \frac{\left(2 s^{\prime}-4 M_{\pi}^{2}\right) \operatorname{Im} F\left(s^{\prime}\right)}{s^{\prime}\left(s^{\prime}-s\right)\left(s^{\prime}-4 M_{\pi}^{2}\right)\left(s^{\prime}+s-4 M_{\pi}^{2}\right)}
$$


By setting $s=2 M_{\pi}^{2}$, and $F=F_{00}$, we find a sum rule important to fix the Adler zeros.

$$
F_{00}\left(4 M_{\pi}^{2}\right)=F_{00}\left(2 M_{\pi}^{2}\right)+\frac{8 M_{\pi}^{4}}{\pi} \int_{4 M_{\pi}^{2}}^{\infty} \mathrm{d} s \frac{\operatorname{Im} F_{00}(s)}{s\left(s-2 M_{\pi}^{2}\right)\left(s-4 M_{\pi}^{2}\right)} .
$$

Finally, for isospin unit exchange, which does not require subtractions,

$$
\operatorname{Re} F^{\left(I_{t}=1\right)}(s, 0)=\frac{2 s-4 M_{\pi}^{2}}{\pi} \text { P.P. } \int_{4 M_{\pi}^{2}}^{\infty} \mathrm{d} s^{\prime} \frac{\operatorname{Im} F^{\left(I_{t}=1\right)}\left(s^{\prime}, 0\right)}{\left(s^{\prime}-s\right)\left(s^{\prime}+s-4 M_{\pi}^{2}\right)},
$$

at threshold this is known as the Olsson sum rule.

Depending on the method we use to fit the S0 wave we find the results in Table 1, where, we have separated on top those fits to data with a total $\chi^{2} /$ d.o.f. $<6$ for the $\pi^{0} \pi^{0}$ and $I_{t}=1$ dispersion relations up to $0.925 \mathrm{GeV}$, a fairly reasonable $\chi^{2} /$ d.o.f. since these fits were obtained independently of the dispersive approach.

However, in Table 1 we also list the very frequently used $t$ and $s$-channel solutions of Estabrooks and Martin [6], those of Protopopescu et al.[5], from Table VI, VIII and table XII, as well as the solution A of Grayer et al. [6]. Their $I_{t}=1$ plus $\pi^{0} \pi^{0}$ dispersion relation total $\chi^{2} /$ d.o.f. is surprisingly poor: $11.3,10.1,7,6,7.5,9.9$, respectively. Therefore, any result that relies heavily on these sets should be taken very cautiously.

We have also improved the previous low energy fits parameters by fitting also the dispersion relations up to $0.925 \mathrm{GeV}$, thus obtaining parameterizations more compatible with analyticity and $s-u$ crossing. Improving from Eq.(4), we find, in $M_{\pi}$ units,

$$
\begin{aligned}
\text { S0; } s^{1 / 2} \leq 2 m_{K}: & B_{0}=17.4 \pm 0.5 ; B_{1}=4.3 \pm 1.4 ; \\
& M_{\mu}=790 \pm 21 \mathrm{MeV} ; z_{0}=195 \mathrm{MeV}[\text { Fixed]; } \\
\text { S2 } ; s^{1 / 2} \leq 1.0: & B_{0}=-80.8 \pm 1.7 ; B_{1}=-77 \pm 5 ; z_{2}=147 \mathrm{MeV}[\text { Fixed]; } \\
\mathrm{P} ; s^{1 / 2} \leq 1.05: & B_{0}=1.064 \pm 0.11 ; B_{1}=0.170 \pm 0.040 ; M_{\rho}=773.6 \pm 0.9 \mathrm{MeV} ; \\
\mathrm{D} 2 ; s^{1 / 2} \leq 1.42: & B_{0}=(2.9 \pm 0.2) \times 10^{3} ; B_{1}=(7.3 \pm 0.8) \times 10^{3} ; \\
& B_{2}=(25.4 \pm 3.6) \times 10^{3} ; \Delta=212 \pm 19 .
\end{aligned}
$$

The D0 and F waves do not change appreciably and we just refer to [3] for details. In Fig. 3 we show the improved curves for S0 and S2, and that of D2 in Fig.1.c.

Concerning the improved fits to individual data sets, we get somewhat different results for S0, listed in Table 2 with the $\chi^{2} /$ d.o.f. of each forward dispersion relation and the standard deviations for the sum rule in Eq.(6) (which are more than four for K decay plus the Grayer B or E or Kaminski improved solutions). For other waves, no matter what S0 fit is used, we find very similar values to those in Eq.(8). This can be checked in Fig.3.b, where we show the improved "K decay + Grayer Sol. B" S2 wave. Even though it is the one for which we obtained the most different central values for the S0 wave compared with those given in Eq.(8), it falls within the uncertainty of our improved solution.

In summary, with forward dispersion relations, we have checked the consistency of different phase shift analyses available in the literature. Surprisingly, some of the most frequently used phase shift sets do not satisfy these dispersive constraints and sum rules, and should therefore be used cautiously. We have provided a simple parameterization of $\pi \pi$ scattering consistent simultaneously with some data sets and all three forward dispersion relations, that we hope could be of use for future studies of $\pi \pi$ scattering. 

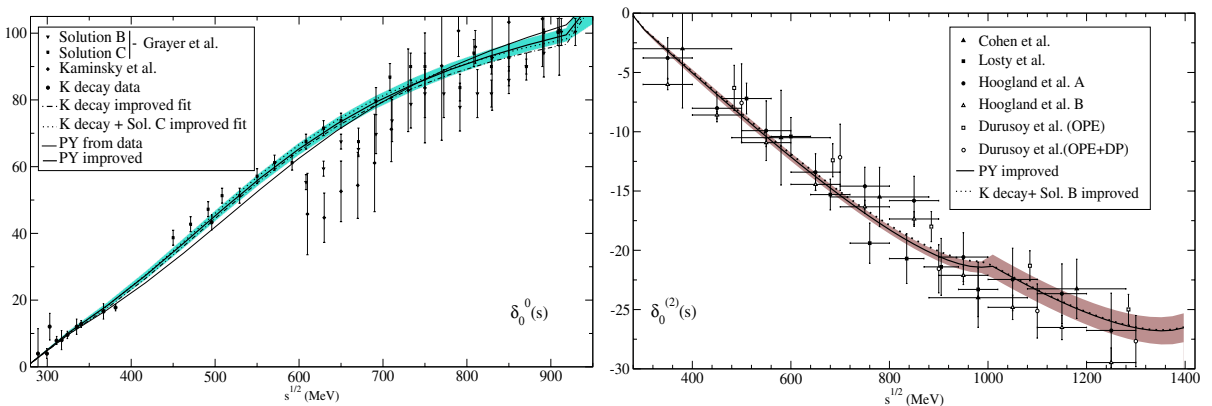

FIGURE 3. a) The improved S0 phase shift (PY improved, Eq.8), the global fit (PY from data, the S0 in Eq. (4)), and the improved solutions " $K$ decay only" and "Grayer $C$ " of Table 2 (almost on top of PY improved). b) S2 improved Phase shift (PY improved, Eq. (8)); global fit (PY from data, Eq. (4)) and the improved parameterization with K decays and So. B of Grayer et al.[6].

TABLE 2. Improved fits (only those already with $\chi^{2} /$ dof $<6$ in Table 1 ). Although errors are given for the Adler zero, we fix it when evaluating other errors, to break the otherwise very large correlations.

\begin{tabular}{|c|c|c|c|c|c|c|}
\hline $\begin{array}{c}\text { Improved } \\
\text { fits: }\end{array}$ & $\begin{array}{c}\text { Improved } \\
\text { PY, Eq.8 }\end{array}$ & $K$ decay only & $\begin{array}{c}K \text { decay+ } \\
\text { Grayer C }\end{array}$ & $\begin{array}{c}K \text { decay+ } \\
\text { Grayer B }\end{array}$ & $\begin{array}{c}K \text { decay+ } \\
\text { Grayer E }\end{array}$ & $\begin{array}{c}K \text { decay+ } \\
\text { Kamiński }\end{array}$ \\
\hline$B_{0}$ & $17.4 \pm 0.5$ & $16.4 \pm 0.9$ & $16.2 \pm 0.7$ & $20.7 \pm 1.0$ & $20.2 \pm 2.2$ & $20.8 \pm 1.4$ \\
$B_{1}$ & $4.3 \pm 1.4$ & $\equiv 0$ & $0.5 \pm 1.8$ & $11.6 \pm 2.6$ & $8.4 \pm 5.2$ & $13.6 \pm 43.7$ \\
$M_{\mu}(\mathrm{MeV})$ & $790 \pm 30$ & $809 \pm 53$ & $788 \pm 9$ & $861 \pm 14$ & $982 \pm 95$ & $798 \pm 17$ \\
$z_{0}(\mathrm{MeV})$ & $195 \pm 30$ & $182 \pm 34$ & $182 \pm 39$ & $233 \pm 30$ & $272 \pm 50$ & $245 \pm 39$ \\
\hline$I_{t}=1, \chi^{2} /$ d.o.f. & 0.40 & 0.30 & 0.37 & 0.37 & 0.60 & 0.43 \\
\hline$\pi^{0} \pi^{0}, \chi^{2} /$ d.o.f. & 0.66 & 0.29 & 0.32 & 0.83 & 0.09 & 1.08 \\
\hline$\pi^{+} \pi^{-}, \chi^{2} /$ d.o.f. & 1.62 & 1.77 & 1.74 & 1.60 & 1.40 & 1.36 \\
\hline Eq.(6) & $1.6 \sigma$ & $1.5 \sigma$ & $1.5 \sigma$ & $4.0 \sigma$ & $6.0 \sigma$ & $4.5 \sigma$ \\
\hline
\end{tabular}

\section{REFERENCES}

1. Peláez, J. R., and Ynduráin, F. J., Phys. Rev. D68, 074005 (2003).

2. Peláez, J. R., and Ynduráin, F. J., Phys. Rev. D69, 114001 (2004).J. R. Pelaez, Int. J. Mod. Phys. A 20, 628 (2005)

3. Peláez, J. R., and Ynduráin, F. J.,Phys. Rev. D71, 074016 (2005).

4. de Trocóniz, J. F., and Ynduráin, F. J., Phys. Rev., D65, 093001, (2002), Phys. Rev. D71, 073008 (2005).

5. Protopopescu, S. D., et al., Phys Rev. D7, 1279, (1973).

6. Cern-Munich experiment: Hyams, B., et al., Nucl. Phys. B64, 134, (1973); Grayer, G., et al., Nucl. Phys. B75, 189, (1974). Estabrooks, P., and Martin, A. D., Nucl. Physics, B79, 301, (1974); Kamiński, R., Lesniak, L, and Rybicki, K., Z. Phys. C74, 79 (1997) and Eur. Phys. J. C4, 4 (2002);

7. Rosselet, L., et al. Phys. Rev. D15, 574 (1977); Pislak, S., et al. Phys. Rev. Lett., 87, 221801 (2001).

8. Losty, M. J., et al. Nucl. Phys., B69, 185 (1974); Hoogland, W., et al. Nucl. Phys., B126, 109 (1977); Cohen, D. et al., Phys. Rev. D7, 661 (1973); Durusoy, N. B., et al., Phys. Lett. B45, 517 (19730.

9. Palou, F. P., and Ynduráin, F. J., Nuovo Cimento, 19A, 245, (1974); Palou, F. P., Sánchez-Gómez, J. L., and Ynduráin, F. J., Z. Phys., A274, 161, (1975).

10. Biswas, N. N., et al., Phys. Rev. Lett., 18, 273 (1967) Cohen, D. et al., Phys. Rev. D7, 661 (1973). Robertson, W. J., Walker, W. D., and Davis, J. L., Phys. Rev. D7, 2554 (1973). Hanlon, J., et al, Phys. Rev. Lett., 37, 967 (1976); Abramowicz, H., et al. Nucl. Phys., B166, 62 (1980). B.G. Zakharov and V.N. Sergeev, Sov. J. Nucl. Phys. 39, 448 (1984) also in Yad. Fiz. 39, 707 (1984). 
Copyright of AIP Conference Proceedings is the property of American Institute of Physics and its content may not be copied or emailed to multiple sites or posted to a listserv without the copyright holder's express written permission. However, users may print, download, or email articles for individual use. 